\title{
Experimental Technology of Operational Pipeline Condition Monitoring
}

\author{
Aleksey Mironov ${ }^{1, a}$, Pavel Doronkin ${ }^{1}$ and Aleksander Priklonsky ${ }^{1}$ \\ ${ }^{1} D$ un $D$ centrs, 7, Margrietas street, Riga, Latvia
}

\begin{abstract}
This work presents a summary of the research study of operational modal analysis (OMA) application for condition monitoring of operating pipelines. Special focus is on the topicality of OMA for definition of the dynamic features of the pipeline (frequencies and mode shapes) in operation. The research was conducted using two operating laboratory models imitated a part of the operating pipeline. The results of finite-element modeling, identification of pipe natural modes and its modification under the influence of virtual failure are discussed. The work considers the results of experimental research of dynamic behavior of the operating pipe models using one of OMA techniques and comparing dynamic properties with the modeled data. The study results demonstrate sensitivity of modal shape parameters to modification of operating pipeline technical state. It is resumed about ability of operating pipeline condition monitoring by measuring dynamic deformations of the operating pipe and OMA techniques application for dynamic properties extraction.
\end{abstract}

\section{Introduction}

Oil and gas operators face problems to keep pipeline safe and workable to avoid adverse effect on environment and to fulfil obligations of product delivery. The same problems operators of urban pipe infrastructures may have, keeping in mind potential hazards to human health. The new approach was proposed for monitoring of most risky parts of operating pipeline, including those that are crossing the railroad or speedway or pass close to residential district. This approach is based on Operational Modal Analysis (OMA) of pipeline dynamic properties applying wide-band deformation sensors.

Since the beginning of the 90's, OMA attracts attention of researchers for dynamic properties study of big civilian objects. OMA techniques allow determining the modal properties of a structure using the system output data only, that is, the dynamic signals of the stressed structure. The system responds to ambient excitation solely and there is no test excitation affecting the system. OMA use some key approaches for evaluation of modal properties, which are distinguished by the methods of data arrangement and processing. OMA application for pipeline condition monitoring is attractive for several reasons:

- the opportunity to receive the dynamic characteristics of the unlimited part of a pipeline;

- modal characteristics are linearized thanks to a wide range of accidental excitations;

- all or some parts of the measured degree of freedom can be used for reference purpose, which significantly increases method resolution and helps to segregate the paired and closely located modes;
- it is useful for the vibration-based health monitoring [1].

Widest OMA application was found for the approaches using conversion in the time domain: Natural Excitation Technique (NExT), model of the general AutoRegression Moving Average vector (ARMA V), Stochastic Subspace-based methods, and approaches in frequency domain, like Frequency Domain Decomposition (FDD) or least-squares complex frequency-domain (LSCF). In this research, the authors considered use of one of OMA approaches - Enhanced Frequency Domain Distribution (EFDD) for evaluation of the condition of a section of an operating pipeline.

This article considers application of OMA technique for damage identification of the experimental model of a pipeline with the actuation by flow inside. Gas or flow streaming inside a pipe excites plenty of structural modes allowing determination of modal properties of surveyed pipeline part. Whereas accelerations typically characterize dynamic behavior of a structure, wall deformations of motionless pipe may be more effective for this purpose. Nowadays, most advanced pipelines already have fiberoptic systems for condition monitoring [2] however, its frequency range is low and they are limited to detect small local damages as joint weld or corrosion spots that cause $50 \%$ of gas pipelines problems as well as other material damages. Being limited by low frequency range, they are not sensitive enough to many important ambient impacts as well. Unlike fiber optics, the film-type piezo-electric sensors distributed along a pipe provide measurement of pipe wall deformations in practically unlimited frequency range. The model of operating pipeline facilitated by

\footnotetext{
a Corresponding author: info@ddcentrs.lv
} 
above deformation measurement system was called smart pipe. The pipe equipped with sensitive deformation transducers works as an antenna that feels any wall deformations caused by streaming flow inside or soil distortion outside. By the way, smart pipe provides adequate dynamic data, required for modal analysis.

The task of discussed research study was to verify the applicability of OMA for damage identification of smart pipe under operating conditions similar to natural ones.

Practical application of modal analysis methods requires identification of natural modes of a structure in determined frequency range, therefore an experimental phase was preceded by mathematical modelling using the finite-element technique (FET).

\section{Modelling}

For modelling a pipe, the triangular parabolic elements evenly spread over the whole surface of the model were used as finite elements (FE). Analysis of the normal modes shape and frequency of oscillation was done in respect to the first 20 natural modes, the displacement being used as a parameter.

Table 1 presents the calculated parameters of the natural modes of a pipeline model and Figure 1 demonstrates principal mode types.

Table 1. Modal frequencies of ideal and "faulty" pipes.

\begin{tabular}{|c|c|c|c|c|}
\hline \multirow[b]{2}{*}{ Mode No } & \multirow{2}{*}{ Mode id } & \multicolumn{3}{|c|}{ Frequency } \\
\hline & & $\begin{array}{l}\text { ideal pipe, } \\
\mathrm{Hz}\end{array}$ & $\begin{array}{l}\text { fault pipe, } \\
\mathrm{Hz}\end{array}$ & $\begin{array}{c}\text { modifi } \\
\text { cation, } \\
\%\end{array}$ \\
\hline $1 \mathrm{a}$ & \multirow{2}{*}{$\begin{array}{c}1^{\text {st }} \\
\text { bending }\end{array}$} & 36.19 & 35.76 & $1 \%$ \\
\hline $1 \mathrm{~b}$ & & 36.19 & 35.79 & $1 \%$ \\
\hline $2 a$ & \multirow{2}{*}{$\begin{array}{c}2^{\text {nd }} \\
\text { bending }\end{array}$} & 219.35 & 212.61 & $3 \%$ \\
\hline $2 b$ & & 219.38 & 214.10 & $2 \%$ \\
\hline 3 & $1^{\text {st }}$ torsion & 487.61 & 478.06 & $2 \%$ \\
\hline $4 a$ & \multirow{2}{*}{$\begin{array}{c}3^{\text {rd }} \\
\text { bending }\end{array}$} & 585.56 & 562.30 & $4 \%$ \\
\hline $4 b$ & & 585.67 & 567.75 & $3 \%$ \\
\hline 5 & $1^{\text {st }}$ longit. & 787.34 & 772.96 & $2 \%$ \\
\hline $6 a$ & \multirow{2}{*}{$\begin{array}{c}4^{\text {th }} \\
\text { bending }\end{array}$} & 1079.65 & 1063.49 & $1 \%$ \\
\hline $6 \mathrm{~b}$ & & 1079.84 & 1064.07 & $1 \%$ \\
\hline $7 \mathrm{a}$ & \multirow{2}{*}{$\begin{array}{l}1^{\text {nd }} \\
\text { shell }\end{array}$} & 1141.86 & 1128.12 & $1 \%$ \\
\hline $7 \mathrm{~b}$ & & 1141.99 & 1128.60 & $1 \%$ \\
\hline $8 \mathrm{a}$ & \multirow{2}{*}{$2^{\text {rd }}$ shell } & 1149.15 & 1134.48 & $1 \%$ \\
\hline $8 b$ & & 1149.18 & 1134.59 & $1 \%$ \\
\hline $9 a$ & \multirow[t]{2}{*}{$3^{\text {th }}$ shell } & 1172.39 & 1159.15 & $1 \%$ \\
\hline $9 \mathrm{~b}$ & & 1172.45 & 1159.60 & $1 \%$ \\
\hline $10 \mathrm{a}$ & \multirow[t]{2}{*}{$4^{\text {th }}$ shell } & 1229.38 & 1215.44 & $1 \%$ \\
\hline $10 \mathrm{~b}$ & & 1229.45 & 1215.55 & $1 \%$ \\
\hline $11 \mathrm{a}$ & \multirow[t]{2}{*}{$5^{\text {th }}$ shell } & 1338.21 & 1325.35 & $1 \%$ \\
\hline $11 \mathrm{~b}$ & & 1338.34 & 1326.02 & $1 \%$ \\
\hline
\end{tabular}

Column ideal pipe (Table 1) contains calculated frequencies of pipe model in ideal (non-faulted) state; column fault pipe includes frequencies of virtually damaged pipe. Analysis showed that the majority of the modes are paired, that is, they "sit" on the closely spaced frequencies and have similar modes with the $90^{\circ}$ phase shift. Paired modes have the same number but differ by letter a or b. Symmetry of the pipe model cross section is the reason of the pairing. Identification of the modes allowed determining of two major mode groups and two isolated modes. The first group includes four pairs of bending modes: 1ab (Figure 1a), 2ab, 4ab, 6ab, under which the pipe behavior is similar to bending oscillations of a beam. Such way of oscillation consider the cross sections of the hollow pipe deform in the same direction in which the longitudinal axis is bending. It means that at vertical bending mode the circular cross section of hollow pipe periodically compresses in vertical direction. Two isolated modes in low-frequency range are extremal cases of beam-like oscillation. The first one (No 3 in Table1, Figure $1 b)$ is interpreted as the $1^{\text {st }}$ mode of torsion oscillations, in which the cross-sections of the pipe swivel around the longitudinal axis. The second one (No 5 in Table1, Figure 1c) is the longitudinal mode, in which the free end of the pipe shifts along the longitudinal axis. In both cases cross sections do not change its round shape but vary diameter.

The second modes group includes five pairs of oscillation modes: 7ab (Figure 1d), 8ab, 9ab (Figure 1e), $10 \mathrm{ab}, 11 \mathrm{ab}$ (Figure 1f), where the wall of the pipe behaves as a shell.

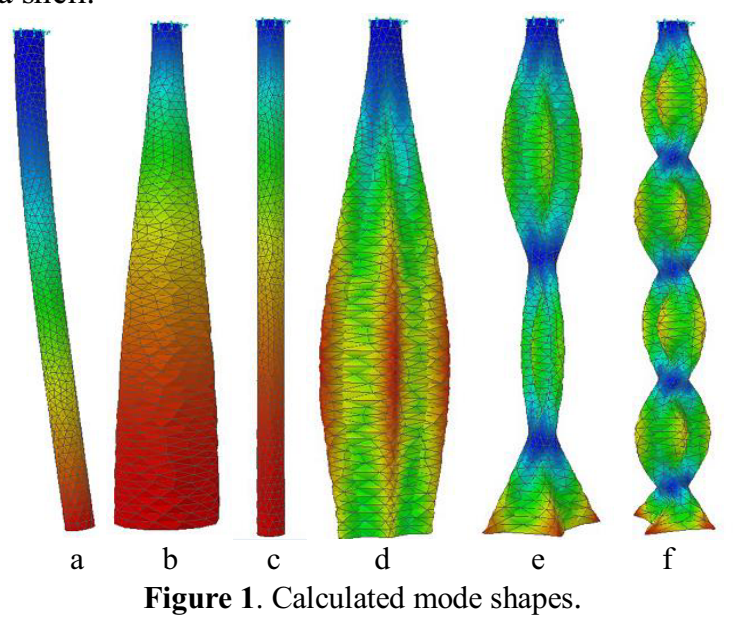

In each of the paired modes, the mode $b$ is similar to mode a, yet turned by $90^{\circ}$. Under shell oscillations the pipe model does not bend as a beam (the pipe axis is not deformed), however, the cross sections get deformed, being "flattened" and "stretched" in perpendicular directions.

To evaluate sensibility of natural modes to the local failure the computational experiment was done, where a defect of a real pipe was modelled as the pipe model wall saw-through (less than 10\% of the circumference and 30\% of the wall thickness).

Analysis of computed modes was limited by numeric evaluation of frequencies only, while evaluation of the mode shapes was done visually. However, since the frequency changing upon introduction of the defect is one of the modal parameters, it can be assumed that the mode is changing along with the change of frequency. Results of the computational experiment are shown in the column faulty pipe of Table 1. Different modes respond to the local 
fault in various ways. Thus, while the frequencies of the paired $1^{\text {st }}$ bending mode, which depends on the global properties of the modelled pipe, decreased by $1 \%$ only, the $2^{\text {nd }}$ and $3^{\text {rd }}$ modes that depend more on the mass and rigidity distribution along the model length decreased up to $4 \%$. For the shell modes, the modal frequencies decreased not more than $1 \%$. Varying response of the modes to a local fault is illustrated by Figure 2 showing the behavior of faulted area along the $3^{\text {rd }}$ bending mode (4ab Table 1) in the two opposite oscillation phases. As is evident, the defect coincides with the maximum deformation spot for this mode, therefore the parameters of that particular mode got distorted to the maximum extent.
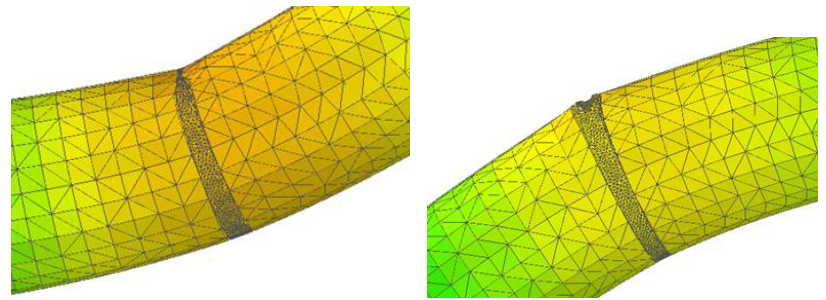

Figure 2. Mode modification after introducing a defect model.

Analysis of numerical pipe model identified two main groups of natural modes: bending and shell ones. Mode shapes of these groups have principal difference and their sensitivity to test fault differs.

\section{Experimental study of operating pipeline model by OMA technique}

The laboratory model of an operating pipeline was equipped with deformation sensors distributed along and circumferentially the pipe. The set of 21 deformation sensors provide data for experimental determination of the dynamic characteristics of a laboratory pipeline model. The laboratory pipeline model has the form of a straight section of a hollow pipe. Turbulent flow of water streaming through the pipe excites its walls. Controlled flow resistance in front of the pipe inlet allows adjusting of excitation level of the pipe walls by modulating the flow turbulence intensity.

Advanced piezoelectric film transducers (Figure 3)

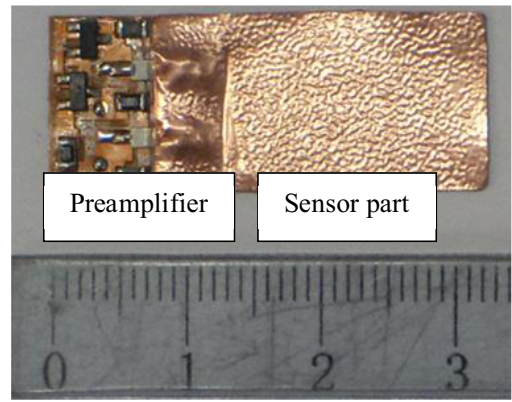

Figure 3. Deformation transducer.

measure dynamic deformations of the oscillating pipe wall. Attached to the pipe surface the ultra-light sensors are exposed to stretching or compression together with the pipe wall almost without affecting the mechanical properties of the pipe. The preamplifier located next to the sensor on a flexible base transforms time-variant charge generated by the sensor under extension and compression of wall into the alternate electric voltage. Combination of the sensor and the preamplifier represents the deformation transducer.

Dynamic range of applied sensors is practically unlimited in terms of both the relative extension or compression and frequency. Tested segment of the experimental pipeline model has 21 transducers in the seven evenly distributed cross sections (Figure 4).

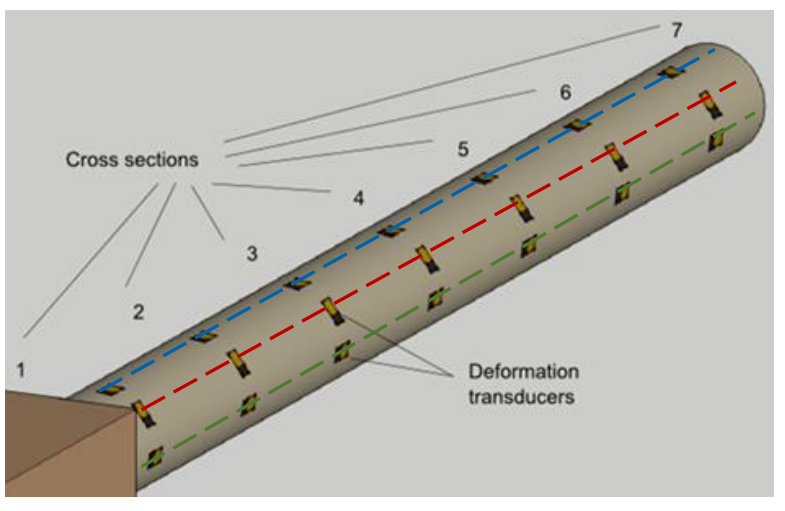

Figure 4. Distribution of transducers on the pipeline segment.

In each cross section, the transducers are located along the pipe axis on three lines that shifted at a $45^{\circ}$ angle from one to another. The transducers located on the top (blue line) and side (green line) generatrix are set by its length along the pipe axis (longitudinal transducers), and their task was to measure extension and compression of the "longitudinal" virtual fibers of the pipe. The transducers on the intermediary generatrix (red line) measured in circumferential direction that means deformation of pipe cross-section (lateral transducers).

Multichannel data acquisition unit collects signals from all 21 transducers simultaneously and then transfers it to the computer that saves the data and performs OMA. The result of data processing is an eigenvector based on geometrical model of the pipeline, which includes the normalized deformation values describing shapes frequencies and damping factors of determined modes.

\subsection{Mode shape identification of one-sided fixed pipe model}

The first version of operating pipeline model was built-up as one-sided fixed steel pipe with measurement set-up as on Figure 4. Diagrams, like presented on Figure 5 , facilitate modes shape identification using experimental measured modal parameters. The normalized deformation magnitudes are related to the cross section number and plotted by taking into account the phase of oscillation (positive or negative). Depending on the location of the data points - the top and side of the pipe (longitudinal transducers) or between them (lateral transducers), the magnitude values on the diagram are connected by a blue, red or green line. Modes identification bases on the assumption that measured deformations of the pipeline model are linked through the second derivate with the 
displacements that were computed for numerical model of the pipeline. Figure 5a illustrates deformation of the pipe model oscillating at $1^{\text {st }}$ bending mode $(21,8 \mathrm{~Hz})$.

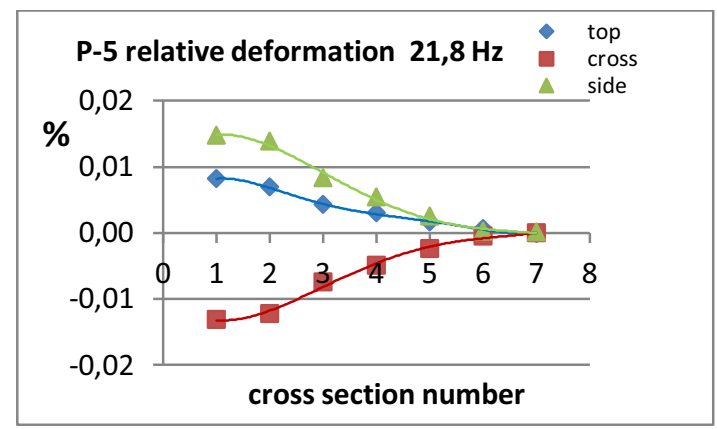

a)

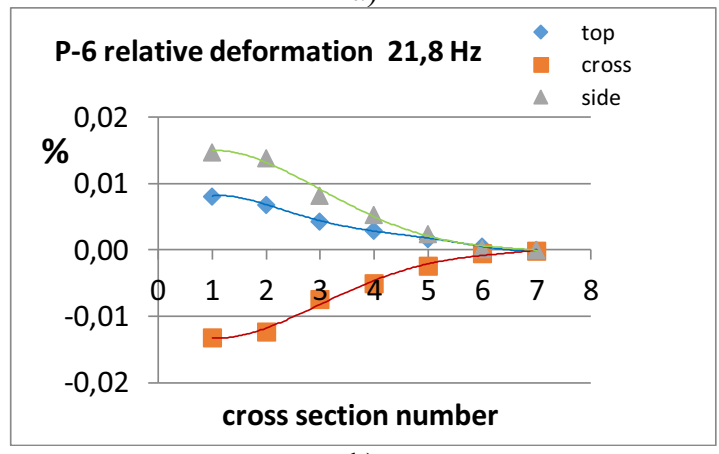

b)

Figure 5. Deformation diagrams of first bending mode: a) initial condition, b) - faulted; top generatrix - blue rhombus; side - green cones; cross generatrix (lateral transducers) - red squads.

The maximum displacement of the free end of the cantilever fitted pipe under the first bending mode is approaching zero deformation that may be seen in the section 7 for all transducers. Conversely, the minimum displacement next to pipe fixity ( $1^{\text {st }}$ section $)$ computed by numerical model is correspondent to the maximum deformation of the pipe walls. Comparison of deformation magnitudes along the top and the side generatrix in the same cross section allows determining the oscillation phase in relation to the fixed coordination system of the laboratory facility. The magnitudes and phase ratio of both lateral sensor lines give an idea about wall deformations distribution along the pipe model. A positive value of the top and the side deformation magnitudes indicates location of oscillation plane between its generatrix. Asymmetrical fixity of the operating pipe model causes $62^{\circ}$ slope of the oscillation plane (under the first bending mode) from the vertical that is why deformations at the side generatrix (green) dominate over the top generatrix (blue) deformations (Figure 5a). Bending of the pipe causes maximal "flattening" of cross sections near its fixity (cross section No 1), so side generatrix (red) appears to be located next to maximal compression line.

Analysis of the experimentally obtained modal shapes demonstrates their similarity to the numerically estimated data. At the same data numerically estimated modal frequencies appear to be lower than experimentally obtained due to simulation problems of experimental model boundary conditions. Thus, the first bending mode computed as $36 \mathrm{~Hz}$, in fact turned out $21.8 \mathrm{~Hz}$. Comparison of calculated and experimentally obtained mode shapes demonstrated their close resemblance, proving the model quality. In analyzing the properties of experimental model through the OMA technique no paired modes predicted by the digital model were revealed. That is because the fixity of the experimental pipe did not correspond to the numerical one.

To estimate the lowest sensitivity limit to mechanical properties modification the local damage was introduced into the laboratory pipeline model. Geometry of the fault was similar to the numerical model (paragraph 2), the cutthrough the wall of the natural pipe was less than $30 \%$ of the wall thickness. Analysis of the experimentally obtained modal parameters of the pipeline model demonstrated variable response of different modes to modification of the technical state (as the numerical analysis also). The deformation distribution diagrams (OMA obtained) reflecting shapes of the first bending mode in the healthy (Figure 5a) and defective conditions (Figure 5b) did not differ practically. Integrated difference of normalized magnitudes between two states of the pipe did not exceed $0.2 \%$, while the frequencies differed by a mere $0.1 \%$, which corresponded to the estimated data.

At the same time, the differences proved more significant in some other modes. For example, the difference of magnitudes for the third bending mode oriented in the inclined plane between the initial (Figure 6a) and defective (Figure 6b) states was: in terms of magnitudes $-12.5 \%$, and in terms of frequency $-0.7 \%$.

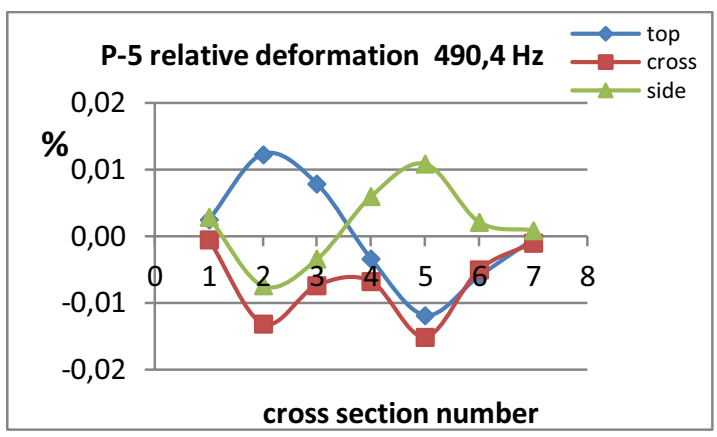

a)

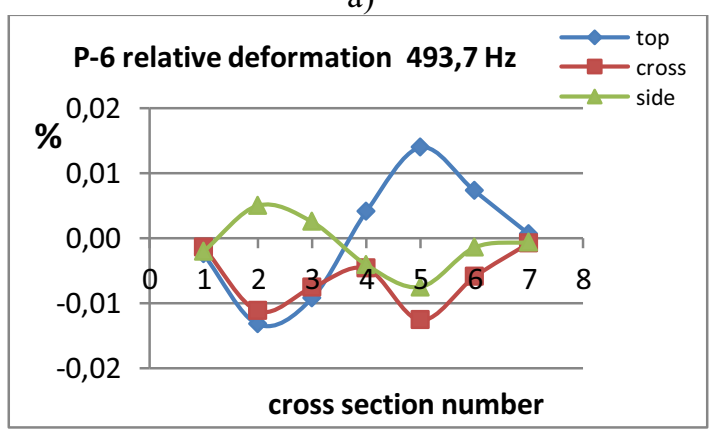

b)

Figure 6. Deformation diagrams of $3^{\text {rd }}$ bending mode: a) - initial condition, b) - faulted; top generatrix - blue rhombus; side green cones; cross generatrix (lateral transducers) - red squads.

Modification of the mode shape happened primarily due to the change of the slope angle of the oscillation plane (in relation to the vertical) from $42^{\circ}$ to $25^{\circ}$ because of the defect. As we may see, the third bending mode has higher response to fault than the first one conditioned on the fact that location of the defect practically coincided with 
position of the maximum deformation for this mode, which was obvious already at the modelling stage.

Considered above means that mode shapes of higher order are more effective for detection of local failures of operating pipeline.

\subsection{Impact of different media and faults to both-sided fixed pipe model}

Another operating pipeline model allowed to study its modal behavior in conditions similar to natural pipeline (Figure 7).

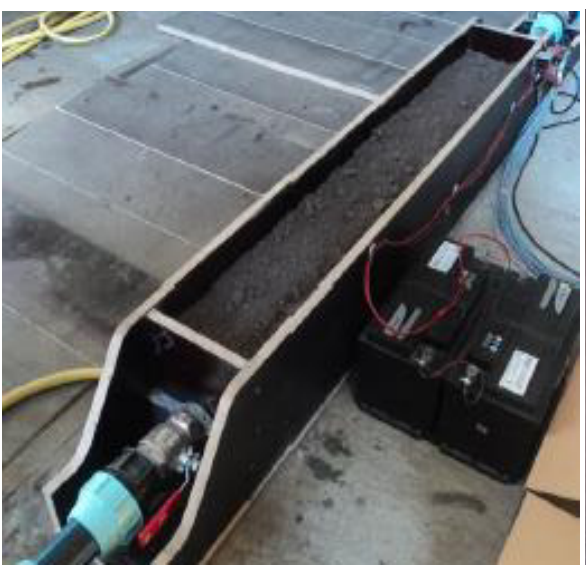

a)

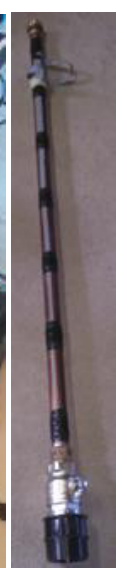

b)
Figure 7. Operating pipeline model: a) - testing box with pipe buried in the soil; b) - the pipe with transducers.

Two opposite walls of the massive box (Figure 7a) are the pillars of the pipe with transducers (Figure $7 b$ ) fixing its ends in the transverse direction and limiting its movement in the axial direction. Both the box and the pipe model with the measurement system are waterproof. Ambient media in the box around the pipe model is variable: pipe can operate in open air, can be buried in a soil, and can be doused in a water. The copper-alloy pipe has the same measurement set-up as described in section 3.1.

As experimental data shows the properties of ambient media may seriously effect on modal parameters of the pipe model. Figure 8 illustrates how varying media modifies the shape of $3^{\text {rd }}$ shell mode of the pipe model in healthy state (no defects). It is evident that more consistent media reduce modal magnitudes slightly (Figure 8a, b, c). Media impact to modal frequency is more effective. For instance, loose soil reduced frequency or $3^{\text {rd }}$ bending mode for $0.2 \%$ only however, water dipping of the operating pipe reduced frequency of the same mode for almost $30 \%$. On top of media impact intensity and composition of the streaming flow may affect modal parameters of pipe model. In case if media outside and flow inside remain stable in a determined time period, the pipe condition monitoring becomes available. For this purpose the parameter of modal parameters variation to be calculated.

The ensemble of estimated modal parameters experimentally obtained using OMA in the state $\mathrm{S}$ of the pipeline model could be written as eigenvector matrix

$$
\mathrm{M}_{\mathrm{S}}=\left[\mathrm{m}_{\mathrm{i}, \mathrm{j}, \mathrm{k}}\right]
$$

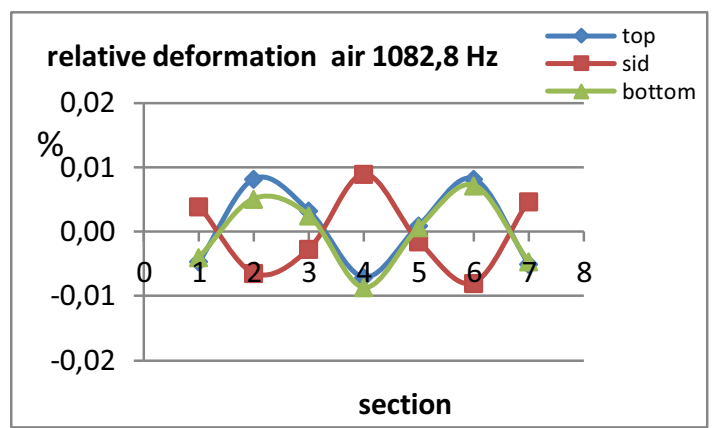

a)

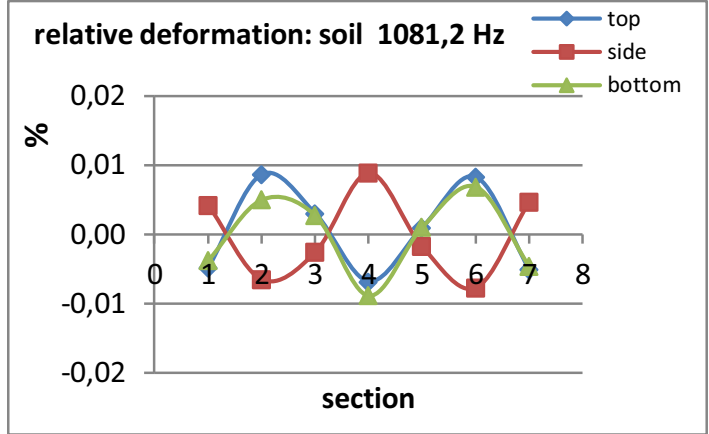

b)

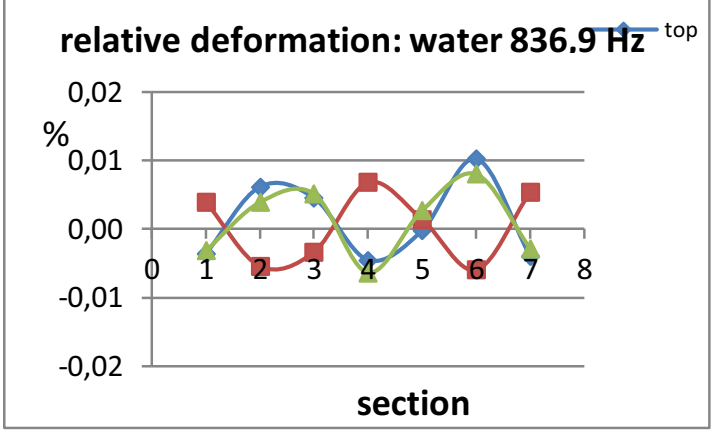

c)

Figure 8. Deformation diagrams of $3^{\text {rd }}$ shell mode with different media: a) - air, b) - soil, c) - water.

where $\mathrm{i}$ - number of DOF in one section (3),

$\mathrm{j}$ - number of sections (7),

$\mathrm{k}$ - mode number,

$m_{i, j, k}$ - eigenvalue measured at $\operatorname{DOF}(i, j)$ of $k$ th mode.

For monitoring purpose current state $\mathrm{S}$ to be compared with initial state (etalon) $\mathrm{E}$ by calculation of its difference

$$
\Delta \mathrm{M}_{\mathrm{S}}=\mathrm{M}_{\mathrm{S}}-\mathrm{M}_{\mathrm{E}}
$$

Modal parameters variation (MPV) estimates difference of modal parameters between current and initial state of the pipe model. Such differential eigenvector for current pipeline state estimates how far this current state of the pipe model is remote from its initial state (or etalon).

Experimental data obtained from testing of five technical states of the operating pipe model provided computation of MPV parameter for:

- healthy or initial state (three tests),

- local fault as thinning of the pipe wall $(20 \%$ of wall thickness and about $0.5 \%$ of square) made by polisher,

- static deflection of the pipe model (maximal deflection $-0,8 \%$ of the pipe model length) simulating earthslide of natural pipeline. 
Figure 9 illustrates variation of mode shapes and frequencies of operating pipe model at the $1^{\text {st }}$ bending mode in above mentioned states.

Symmetry of diagram of healthy state (figure 9a) is broken by green line of side sensors line distortion (Figure 9b) when pipe wall was thinned locally. Maximal distortion of diagram red line locates close to the place, where the fault was implemented. Diagrams of Figure 9c dramatically change in comparison with healthy state (Figure 9a) as well as the frequency dropped for $10 \%$. The reason is that even slightly deflected pipe has lost symmetry and as the result has greatly modified its mechanical properties. Analysis of found mode shapes of the pipe model shows that MPV parameters have various sensitivity of bending and shell mode shapes to different faults. For instance, MPV of bending modes is more sensitive to static curve of the pipe than shell ones. However, sensitivity of MPV to local faults depends mostly of fault types and mode orders. It means, there is no privilege for MPV of mode types for detection of local faults.

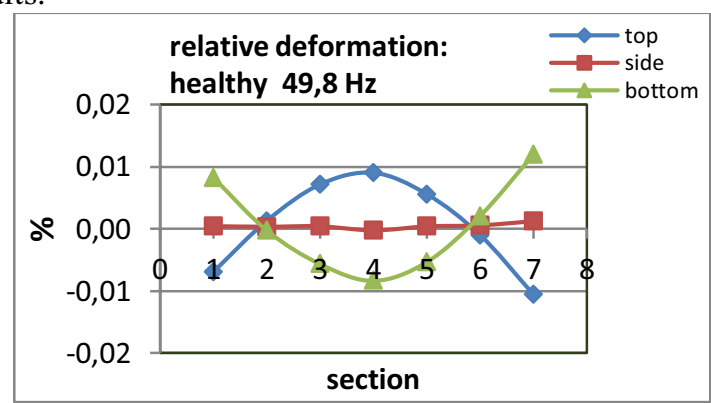

a)

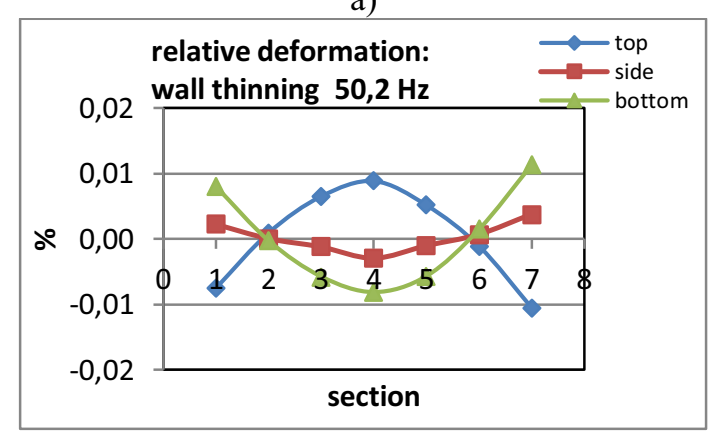

b)

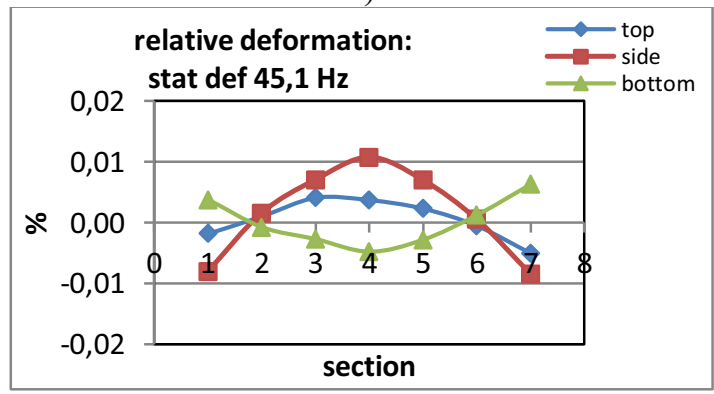

c)

Figure 9. Deformation diagrams of the $1^{\text {st }}$ bending mode in: a) - healthy state, b) - wall thinning, c) - static deflection.

As illustration of MPV sensitivity in depend of mode type Figure 10 shows MPV histogram for three pipe technical states. For both types of modes the scatter of MPV does not exceed 2.2..2.4dB within "healthy" states of operating pipe. Also in case of local fault (thinning)
MPV for both modes grows to $4 \mathrm{~dB}$ and more. However, static curve of operating pipe increase MPV of only bending modes (to $6.6 \mathrm{~dB}$ ), whereas shell modes parameter raises less (up to $4.3 \mathrm{~dB}$ ).

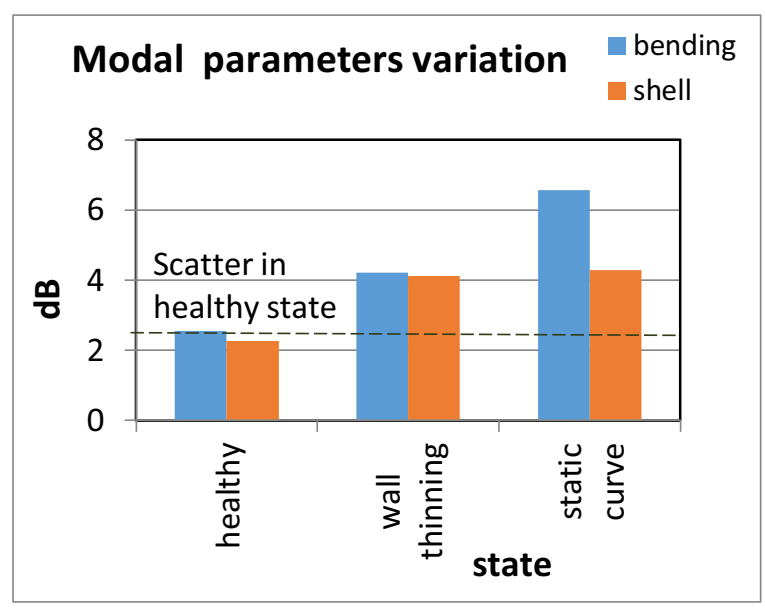

Figure 10. Modal parameters variation in depend of the pipe model technical state

Results of OMA trial application for condition assessment of the experimental pipeline demonstrates varying sensitivity of the modal parameters to defects. While the frequencies of the natural modes changed less, modification of the pipe deformation shapes was quite obvious. That happened because the frequency of every mode is a global parameter of an object, while the deformation distribution (mode shapes) is the function of the local mass and stiffness distribution of the structure. Hence, the diagnostic efficiency of the modal shape parameters is much higher in comparison to the mode frequency parameters. This explains the fact that changes in the shape of the higher order modes as a response to a defect are able to become a reliable indicator of the alterations - even a relatively smaller-scale local changes of the structural state.

\section{Conclusions}

Finite-element modelling proved applicability of modal properties for condition monitoring of operating pipeline. Experimental model of operating pipeline of laboratory scale was built up for OMA techniques validation as the tool for pipe condition monitoring. Turbulent flow stream excited pipeline model walls provides ambient excitation required for OMA application. The measurement system including experimental piezo-electric deformation transducers and 21- channel acquisition unit characterized dynamic behavior of the pipeline functional model. The EFDD technique was applied to compute parameters of natural modes using experimentally obtained data. Numerically computed modes provide identification of experimentally obtained modes that ensures reliable estimation of the changes in the modal parameters. It was revealed that using the mode shape parameters as the diagnostic indicators is more advantageous compared to mode frequencies. It was proved that the modal parameters of the 
operating pipeline obtained using modal analysis technique are able to characterize its dynamic properties, so modification of these properties could be used as diagnostic indicators of pipeline model defects. Thus, the research study of the pipeline model has proven ability of operating pipeline condition monitoring applying OMA techniques to dynamic deformations measured on the operating pipe.

\section{Acknowledgements}

The paper uses materials related to research study No. 1.22 project of the project „Establishment of Transport Mechanical Engineering Competence Center" in cooperation with Investment and Development Agency of Latvia (L-KC-11-0002).

\section{References}

[1] L. Zhang, R. Brincker and P. Andersen, An Overview of Operational Modal Analysis: Major Development and Issues, in: Proc. 1st International Operational Modal Analysis Conference, Copenhagen, Denmark, 2005.

[2] D. Inaudi, B. Glisic, Long-Range Pipeline Monitoring by Distributed Fiber Optic Sensing, Journal of Pressure Vessel Technology, February 2010, vol. 132 / 011701-9 